\title{
Uterine Corpus Cancer by AJCC v7 Stage
}

National Cancer Institute

\section{Source}

National Cancer Institute. Uterine Corpus Cancer by A/CC v7 Stage. NCI Thesaurus. Code C91218.

A term that refers to the staging of uterine corpus cancer (carcinoma or carcinosarcoma) according to the American Joint Committee on Cancer, 7th edition. 